\title{
Real-Time Dynamics of a Spin Chain with Dzyaloshinskii-Moriya Interactions: Spiral Formation and Quantum Spin Oscillations
}

\author{
E. Solano-Carrillo, R. Franco, and J. Silva-Valencia \\ Departamento de Física, Universidad Nacional de Colombia, A.A. 5997, Bogotá-Colombia.
}

(Dated: April 20, 2022)

\begin{abstract}
We studied the non-equilibrium short-time dynamics of a spin-1/2 chain with DzyaloshinskiiMoriya interactions after a sudden quench by a transverse field. We found that inhomogeneous spin-spirals with opposite chiralities propagate from the edges towards the center of the chain. This propagation is accompanied by quantum spin oscillations which decay asymptotically with time. A theoretical description of this phenomenon is given to a good accuracy with the help of numerical calculations with the adaptive time-dependent density matrix renormalization group algorithm.
\end{abstract}

\section{INTRODUCTION}

In recent years, many attempts have been made to understand the non-equilibrium dynamics of closed interacting quantum systems $\stackrel{1}{-1}$. These efforts are mainly motivated by the high degree of isolation and control of microscopic parameters which can be achieved experimentally in systems such as ultracold atoms trapped in optical lattices ${ }^{2}$, which are currently being used to "engineer" quantum spin systems with desired properties ${ }^{3}-\underline{5}$ with the aim of simulating the behavior of emblematic systems in quantum statistical physics and discovering potential technological applications. Among the most studied problems which have been addressed, the dynamics of low-dimensional systems following a quantum quench has received special attention. This is one of the simplest ways to drive a system far from equilibrium. It consists of preparing the system in the ground state of an initial Hamiltonian and then suddenly (or slowly) changing the interactions at a given time, by varying an experimentally tunable parameter, with the result of letting the system evolve unitarily under a final Hamiltonian. As an example, one might mention the quenching dynamics by a transverse magnetic field (tunable parameter) in systems like the XY chain ${ }^{6}-\underline{9}$ or the quantum Ising chain ${ }^{10}-14$, which can be simulated in ultracold atomic systems 5 .

Quenching by a transverse field is a typical protocol for studying non-equilibrium dynamics, since apart from being easy to implement in real situations, it allows the possibility of taking the system (in some cases) through a quantum phase transition, involving macroscopic changes in the many-body state at the final point, as in the quantum Ising chain ${ }^{\underline{5}}$. In more general quenching scenarios, the universal properties of the dynamics near quantum critical points as well as in generic gapped and gapless systems have been investigated thoroughly ${ }^{1}$; in some cases it is possible to make universal predictions of scaling laws for relevant physical quantities. Also, the question of whether the system relaxes to a quasistationary state in the long-time limit after the quench and, if it does, how to characterize its physical properties, has been addressed with a great interest. In particular, the concept of thermalization of a quantum many-body system following a quench has acquired importance; it has been conjectured that the asymptotic state can be described by a generalized Gibbs ensemble ${ }^{15,16}$ if the system is integrable, and by some effective thermal distribution in the case of a generic system. Another main line of research, which we follow in this paper, is to study the characteristics of the time evolution just after the quench (short-time dynamics). This is of special interest, for example, in quantum computation scenarios ${ }^{17}$ in which gate operations require a precise knowledge of the immediate dynamical response of the system. Experimental examples following this line of research are the oscillations $\frac{18}{18}$ or the dephasing 19 of the matter wave field of a Bose-Einstein condensate, features which are also observed in the antiferromagnetic order parameter of a system of ultracold atoms in an optical lattice with superexchange interactions $\stackrel{4}{ }$.

There are many spin systems which serve as prototypes for studying the short-time non-equilibrium dynamics following a quantum quench. In this paper we study a spin system with exchange-relativistic interactions which, according to our knowledge, has not been investigated so far in this context. These kind of interactions arise when Anderson's superexchange mechanism is extended to include spin-orbit coupling. As is well known, the spin-orbit interaction (which couples the spin to the crystal lattice, giving rise to easy and hard magnetization axes) is gaining considerable attention due to the possibility of manipulating spins in solid state systems solely by electric fields 20 and/or perhaps by applied local strain 21,22 . In low-dimensional systems, the lack of structural inversion symmetry which is induced by surfaces and interfaces, combined with the spin-orbit coupling leads to anisotropic antisymmetric superexchange or Dzyaloshinskii-Moriya interaction (DMI) ${ }^{23,24}$, which is responsible for the presence of weak ferromagnetism in a variety of antiferromagnetic compounds 24 . One of the most striking consequences of this spin interaction is the appearance of homochiral spin spiral magnetic structures, as have been observed recently along fixed crystallographic directions (easy-axis directions in some cases) in ferromagnetic and antiferromagnetic ultrathin films using spin-polarized scanning tunneling microscopy ${ }^{25-27}$. These one-dimensional spin structures have been postu- 
lated to play a crucial role in spintronic devices, since spin-polarized currents flowing through them will exert a spin-torque on the chiral magnetic structure, causing a variety of controllable excitations 25 . They are also the most likely candidates to host ferroelectricity, according to recent experimental results ${ }^{28}-30$. In the context of ultracold atoms in optical lattices, it remains to be discovered whether the DMI can be artificially implemented and controlled, as can be done with the symmetric superexchange interaction ${ }^{4}$. Due to the great interest in the consequences of this antisymmetric interaction, this is expected to be achieved soon, since the spin-orbit coupling has already been demonstrated recently in these systems of atoms 31 .

Motivated by the above-mentioned potential applications as well as by the search for fundamental knowledge, in this paper we study the short-time non-equilibrium dynamics in the simplest system with DMI: a spin- $1 / 2$ chain, after a quantum quench is carried out by means of a transverse field. To be more specific, we consider a quantum quench which consists of preparing the initial state $\left|\psi_{0}\right\rangle=|\rightarrow \rightarrow \cdots \rightarrow\rangle$ by applying a strong magnetic field along the $x$-direction, and then turning it off abruptly at $t=0$. The subsequent unitary evolution, $\left|\psi_{t}\right\rangle=\exp (-i \mathcal{H} t)\left|\psi_{0}\right\rangle$, is governed by the final Hamiltonian (we set $\hbar=1$ throughout this paper)

$$
\begin{aligned}
\mathcal{H}=\sum_{j}[ & D\left(S_{j}^{x} S_{j+1}^{y}-S_{j}^{y} S_{j+1}^{x}\right) \\
& \left.+J\left(S_{j}^{x} S_{j+1}^{x}+S_{j}^{y} S_{j+1}^{y}+\Delta S_{j}^{z} S_{j+1}^{z}\right)\right],
\end{aligned}
$$

where the usual DMI in the $z$-direction is taken together with the Heisenberg-type symmetric exchange coupling (EC) with anisotropy $\Delta$ (also known as the XXZ exchange interaction). As an example of a real quasi-onedimensional compound described by this Hamiltonian we mention $\mathrm{KCuF}_{3}$, which is an antiferromagnetic spin- $1 / 2$ system with DMI and almost isotropic EC $\underline{32}$. The critical behavior of this system will be briefly discussed in section [II. Meanwhile, we simply mention that the DMI makes the phase diagram rich, with two critical points (as in the XXZ model) dependent on $D$, and restores the otherwise spoiled ground-state entanglement due to anisotropy (which tends to align the spins), by means of enhanced quantum fluctuations ${ }^{33}$. With respect to some dynamical and thermal features, this model has attracted attention in the quantum information community; for instance, the striking phenomenon of finite-time disentanglement ${ }^{34}$ has been predicted for the two-qubit system prepared in the Werner state $\frac{35}{}$, and teleportation of states has been studied using the model in thermal equilibrium as a quantum channel ${ }^{36}$.

In most of the numerical calculations in this paper, we use the adaptive time-dependent density matrix renormalization group algorithm (t-DMRG) $\stackrel{37.38}{ }$, which has proved to be a powerful method for studying the dynamics of one-dimensional quantum many-body systems with short-ranged interactions. As a general result, we show that the DMI gives rise to inhomogeneous spinspirals with opposite chiralities, which propagate from the edges towards the center of the chain until they meet each other, and then complex interference patterns arise. The propagation is accompanied by quantum spin oscillations that decay asymptotically with time. It is the aim of this work to theoretically describe these oscillations, as well as the nature of the propagation of the spin-spirals. The paper is organized as follows: in section $\amalg$ we study in detail the role played only by the DMI in the formation of these spirals. For this, we use results from the two-spin quantum dynamics and many-body classical dynamics to interpret the observations from the quantum model. In section III we add the exchange interactions and investigate how the results from section $\Pi$ are modified. In section IV, we make some comments about the long-time dynamics in our system; and finally in section [ $]$ we give the conclusions.

\section{EFFECTS OF THE DMI}

Although the DMI is usually expected to be negligible (due to its relativistic nature) compared to the EC, there are instances $\frac{25,26}{2}$ where it is strong enough $(D / J \sim 0.25)$ to create remarkable spin-spiral structures. In this section, we take the limit $J=0$ in order to investigate the effects on the dynamics due only to the DMI. Then Eq. (11) is reduced to

$$
\mathcal{H}^{\mathrm{DM}}=D \sum_{j}\left(S_{j}^{x} S_{j+1}^{y}-S_{j}^{y} S_{j+1}^{x}\right) .
$$

This model is exactly solvable, since it can be mapped onto the XX model: $\mathcal{H}^{\mathrm{XX}}=D \sum_{j}\left(\tilde{S}_{j}^{x} \tilde{S}_{j+1}^{x}+\tilde{S}_{j}^{y} \tilde{S}_{j+1}^{y}\right)$ (which is well known to be equivalent to a set of free spinless fermions) by rotating the basis at each site $j$ by an angle $j \frac{\pi}{2}$ around the $z$-axis. Moreover, its eigenstates are non-equilibrium or spin-current-carrying stationary states of the XX model at zero temperature, since the macroscopic spin-current $J^{M}=\sum_{j} J_{j}^{M}$ (quantized along the direction of $D$ ) defined through the continuity equation

$$
\dot{S}_{j}^{z}=i\left[\mathcal{H}^{\mathrm{XX}}, S_{j}^{z}\right]=J_{j}^{M}-J_{j-1}^{M},
$$

can be found 39 to give $J^{M}=\mathcal{H}^{\mathrm{DM}}$ and, given that this spin-current is a conserved quantity in the XX model, it follows that $\left[\mathcal{H}^{\mathrm{XX}}, \mathcal{H}^{\mathrm{DM}}\right]=0$. The time evolution resulting from several initial states in the XX model such as the domain-wall state at the center of the chain ${ }^{39}-\underline{41}$, the Néel state and the spin-density wave state $\underline{42}$ has already been solved analytically, yielding a variety of phenomena such as quantum fronts in which quantized steps of spin flips are transported and oscillatory dynamics of the order parameter. Our initial spin-polarized state in the $x$-direction $\left|\psi_{0}\right\rangle \propto(|\uparrow\rangle+|\downarrow\rangle) \otimes(|\uparrow\rangle+|\downarrow\rangle) \cdots \otimes(|\uparrow\rangle+|\downarrow\rangle)$ contains eigenstates of the XX model such as the fully polarized states $|\uparrow \uparrow \cdots \uparrow\rangle$ and $|\downarrow \downarrow \cdots \downarrow\rangle$, and also many 


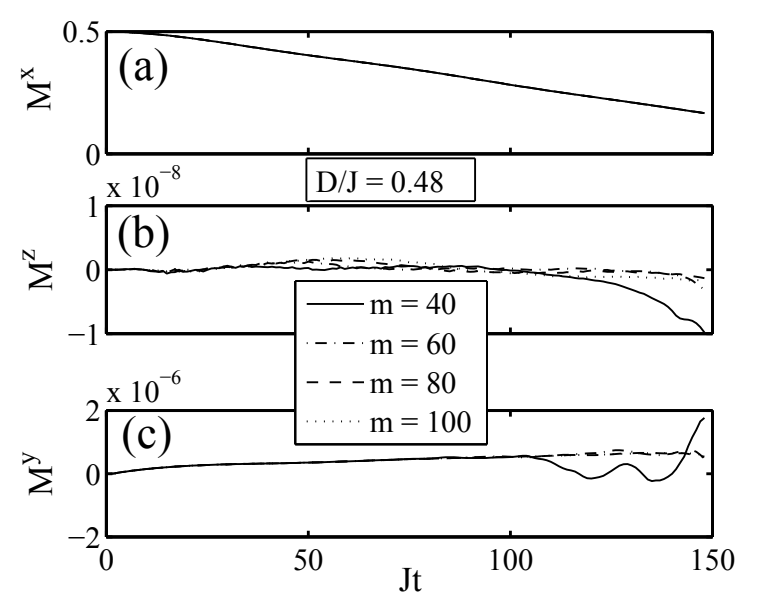

FIG. 1. Time evolution of the components of magnetization for several values of the t-DMRG truncation parameter $m$ in the arbitrary case with DMI and EC for which $D / J=0.48$. (a) $x$-component. (b) $z$-component. (c) $y$-component.

excitations with a large number of domain walls (the Néel state being one of those); thus it is a highly nonequilibrium state at zero temperature. When this initial state is evolved under the XX Hamiltonian, there is no signature of dynamical formation of spin-spirals. Otherwise, when it is evolved under the DM Hamiltonian, the trajectory in the Hilbert space of the resulting state is different, and the formation of the spin-spirals is observed. Since there are no driving fields to excite this wave phenomenon, the open boundary conditions in our finite system play a crucial role in the establishment of these spin-currents. In particular, since $\left\langle\psi_{0}\left|J^{M}\right| \psi_{0}\right\rangle=0$, we expect to encounter a positive spin-current propagating to the right from one boundary, and a negative spincurrent propagating to the left from the other boundary. Although in principle the real-time evolution can be investigated analytically in this case, it is a highly nontrivial problem; therefore we use the t-DMRG algorithm to inquire further into this.

\section{A. Many-body quantum dynamics}

We have used the t-DMRG algorithm for a chain with $N=200$ sites, with a Trotter time step $\delta t=0.05$, and keeping $m=40-100$ density matrix eigenstates to represent the truncated sector of the evolved wave function. To test the convenience of this choice of simulation parameters, we show in Fig. 11 the time evolution of the magnetization $M^{\alpha}=(1 / N) \sum_{j}\left\langle S_{j}^{\alpha}\right\rangle(\alpha=x, y, z)$ for several values of $m$, in an arbitrary case with $J \neq 0$ in which strong long-ranged correlations are expected to lower the accuracy of the algorithm compared to the case $J=0$. As can be seen, for values $m \geq 60$, the $m$-dependent error in the chosen time window is of order $10^{-7}$ in the worst case, which is a satisfactory error for DMRG users. In the following calculations, we used $m=60$ (unless oth-

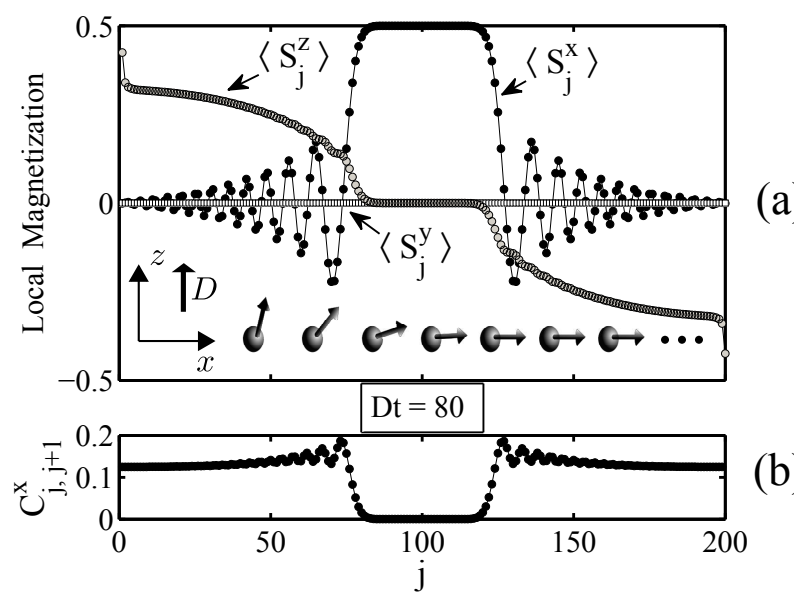

FIG. 2. Snapshot of the local magnetization and nearestneighbor spin-spin correlations in the $x$-direction at the arbitrary time $D t=80$. (a) As can be seen, inhomogeneous spinspirals with opposite chiralities propagate, in the $x z$-plane, from the edges towards the center of the chain, accompanied by spin oscillations. A classical picture of the left-rotating spiral arising from the left end is drawn when $D t=2$ (only the directions of spins are taken into account). (b) The spirals propagate with the same velocity with which nearest-neighbor correlations move.

erwise stated) with the aim of saving computer resources and speeding them up. Note that when the value of $J$ is not shown in the figures, it is assumed to be zero.

Returning to the case of relevance for this section $(J=0)$, we show in Fig. 2] "snapshot" of the local magnetization along the chain as well as the nearest-neighbor spin-spin correlations $C_{j, j+1}^{x}=\left\langle S_{j}^{x} S_{j+1}^{x}\right\rangle-\left\langle S_{j}^{x}\right\rangle\left\langle S_{j+1}^{x}\right\rangle$ in the direction of the polarization of the initial state, at the arbitrary time $D t=80$. As can be seen, inhomogeneous spin-spirals with opposite chiralities propagate (in the $x z$-plane) from the edges towards the center of the chain, as expected from the spin-current considerations given above. This propagation is accompanied by spin oscillations around the $z$-axis. A sketch of the left-rotating spiral propagating from the left end of the chain is drawn when $D t=2$. We verified that the chirality (sense of rotation) of the spin-spirals reverses when the sign of $D$ is reversed, which is an inherent property of the $\mathrm{DMI}^{27}$. We also followed the wave fronts, and found a constant velocity of propagation $v=1$ (in units of $a D, a$ being the lattice constant). As can be seen in Fig. 2(b), this is the same velocity with which nearest-neighbor correlations propagate from the edges, in accordance with the light-cone propagation which is expected for spin models with sufficiently local interactions 43,44 .

It is also interesting to investigate the time evolution of long-ranged correlations, which we calculate as is usually done in the DMRG framework ${ }^{45,46} C_{r}^{x}=\left\langle S_{i}^{x} S_{j}^{x}\right\rangle-$ $\left\langle S_{i}^{x}\right\rangle\left\langle S_{j}^{x}\right\rangle$, where $i=N / 2+1-r$ and $j=N / 2+r$ (when $r$ is increased, $i$ and $j$ move outwards symmetrically from the center towards the edges of the chain). We found 
that long-ranged correlations are quite small in this case: they are always below $\mathrm{O}\left(10^{-3}\right)$ during the spiral propagation, compared to the nearest-neighbor ones which, as may be seen in Fig. 2(b), are $\mathrm{O}\left(10^{-1}\right)$; this is why they are not shown. We shall see later that when $J \neq 0$, strong long-ranged correlations develop throughout the system, making the dynamics more intricate. Meanwhile, let us first try to understand the nature of the results obtained so far.

\section{B. Two-spin quantum dynamics}

We begin by considering the quantum dynamics of a two-spin system, which should contain relevant information about the behavior of a pair of nearest-neighbor sites in the many-body system. In order to simplify the description of states, we rotate the basis temporarily by changing $x \leftrightarrow z$ in Eq. (1), which leads to a DMI in the $x$-direction and a fully polarized initial state in the $z$-direction. Note that this operation does not change expected values of observables. It is also convenient to introduce the Bell states in this new representation, defined as $|t / s\rangle=(|\uparrow \downarrow\rangle \pm|\downarrow \uparrow\rangle) / \sqrt{2}$ and $|\bar{t} / \bar{s}\rangle=(|\uparrow \uparrow\rangle \pm|\downarrow \downarrow\rangle) / \sqrt{2}$. Then we show in Fig. B(a) the energy levels and the stationary states of the two-spin model, with $\omega_{R}=D / 2$. Energetically speaking, this is equivalent to a two-level magnetic system with a spinless intermediate state. In the present case $(J=0)$, when the initial state $\left|\psi_{0}\right\rangle=|\uparrow \uparrow\rangle$ is prepared, the evolved two-spin state reads

$$
\left|\psi_{t}\right\rangle_{2 s}=\frac{1}{\sqrt{2}}\left[|\bar{t}\rangle+\cos \left(\omega_{R} t\right)|\bar{s}\rangle-\sin \left(\omega_{R} t\right)|s\rangle\right],
$$

which shows Rabi oscillations between the states $|\uparrow \uparrow\rangle$ and $|\downarrow \downarrow\rangle$, i.e., the $z$-components of local magnetization for the spin pair oscillate in phase $\left\langle S_{1}^{z}\right\rangle=\left\langle S_{2}^{z}\right\rangle=\frac{1}{2} \cos \left(\omega_{R} t\right)$ with the Rabi frequency $\omega_{R}$. Remarkably, the most probable state to find after an energy measurement of the spin pair (during a cycle) is the spinless intermediate Bell state $|\bar{t}\rangle$, so, at any time, spin measurements in the direction perpendicular to $D$ will disperse around zero, as indeed is seen in Fig. 2(a).

Returning to the original representation, we have a two-spin system with DMI in the $z$-direction and initially prepared in the state $\left|\psi_{0}\right\rangle=|\rightarrow \rightarrow\rangle$. The time evolution shows Rabi oscillations between the states $|\rightarrow \rightarrow\rangle$ and $|\leftarrow \leftarrow\rangle$ with $\left\langle S_{1}^{x}\right\rangle=\left\langle S_{2}^{x}\right\rangle=\frac{1}{2} \cos \left(\omega_{R} t\right)$. A natural question then arises: how can we generalize these results to many sites? It is reasonable to assume that, at least for times $D t<1$, the evolved state is close to

$$
\left|\psi_{t}\right\rangle \stackrel{?}{\sim}\left|\psi_{t}^{+}\right\rangle_{2 s} \otimes|\rightarrow \rightarrow\rangle \otimes \cdots \otimes|\rightarrow \rightarrow\rangle \otimes\left|\psi_{t}^{-}\right\rangle_{2 s},
$$

where $\left|\psi_{t}^{+}\right\rangle_{2 s}$ is the state in Eq. (4) written in the original representation and $\left|\psi_{t}^{-}\right\rangle_{2 s}$ is the corresponding state obtained when changing $D \leftrightarrow-D$, in order to account for the opposite chirality of the spiral arising from the right

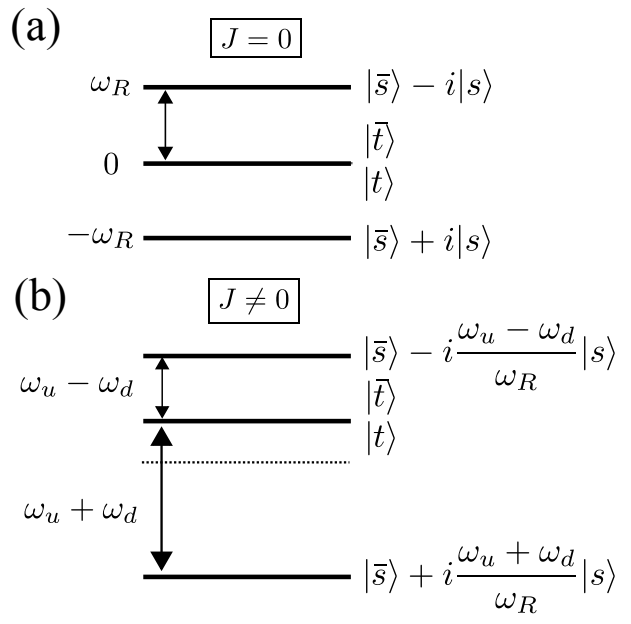

FIG. 3. Energy levels and stationary states of the two-spin model with DMI. (a) In the absence of EC, the first excited state is two-fold degenerate. (b) In the presence of EC, the first excited state remains degenerate, but the energy gaps are modified.

end of the chain (negative spin-current). This assumption is motivated by the fact that for $D t<1$, spin-spin correlations have not crossed the boundaries of the twosite blocks at the ends of the chain, so the state can be thought of as factorized into "non-interacting dimers": $\bullet \bullet \bullet \bullet \cdots \bullet \bullet \bullet \bullet \bullet$ ・ i.e., a product of twospin solutions. The problem with this assumption is that it does not take into account the spiral propagation; i.e., since the perturbations originated at the chain boundaries propagate with a finite velocity, it is clear that the spins within these dimers must oscillate with different phases, so the state in Eq. (5) should be modified to accomplish this.

A convenient way to account for the spiral formation is to decouple the two-spin dynamics by introducing the reduced density matrix $\rho_{t}^{ \pm}=\operatorname{Tr}_{2}\left|\psi_{t}^{ \pm}\right\rangle_{2 s}\left\langle\psi_{t}^{ \pm}\right|$for the spins that have been reached by the perturbations, and $\rho_{0}=\mid \rightarrow$ \rangle$\langle\rightarrow|$ for those that have not. Then, an easy calculation leads to $\rho_{t}^{ \pm}=\frac{1}{2} \mathcal{I}+\cos \left(\omega_{R} t\right) S^{x} \pm \frac{1}{2} \sin (D t) S^{z}$ and $\rho_{0}=$ $\frac{1}{2} \mathcal{I}+S^{x}$, where $\mathcal{I}$ is the $2 \times 2$ identity matrix. Note that this decoupling of the two-spin dynamics preserves the relation $\left\langle S^{x}\right\rangle=\operatorname{Tr}\left(\rho_{t}^{ \pm} S^{x}\right)=\frac{1}{2} \cos \left(\omega_{R} t\right)$. With this, we expect that the evolved state will be close to

$$
\rho_{t} \sim \bigotimes_{j=1}^{N / 2} \Lambda_{j}^{+}(t) \bigotimes_{j=N / 2+1}^{N} \Lambda_{j}^{-}(t),
$$

where $\Lambda_{j}^{ \pm}(t)=\left[1-H\left(\tau_{j}\right)\right] \rho_{0}+H\left(\tau_{j}\right) \rho_{\tau_{j}}^{ \pm}$selects the approximate state of the site $j$ at time $t$, with $H$ being the Heaviside step function and $\tau_{j}=t-(j-1) / D\left(\tau_{j}=0\right.$ the moment at which the perturbation hits the site $j$, measured from the chain boundaries). We tested the validity of the ansatz in Eq. (6) by calculating the exact time evolution of local magnetization for a system with 14 sites,

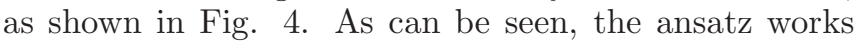




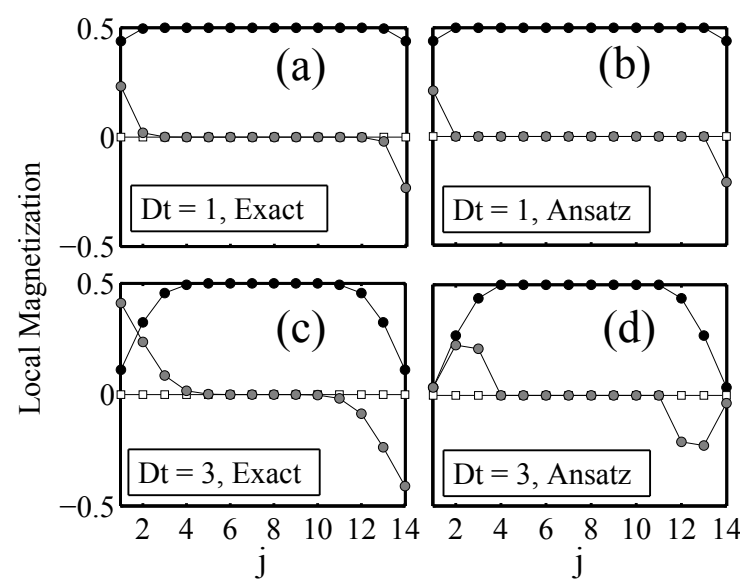

FIG. 4. Snapshots of the local magnetization for a system with 14 sites, calculated exactly: (a), (c); and with the ansatz in Eq. (6): (b), (d), for two different times. The black-filled circles are values of $\left\langle S_{j}^{x}\right\rangle$, the gray-filled ones are those of $\left\langle S_{j}^{z}\right\rangle$, and the white-filled squares are values of $\left\langle S_{j}^{y}\right\rangle$, as in Fig. 2(a). Solid lines are guides for the eyes.

very well for times $D t \leq 1$ as expected from the arguments motivating Eq. (5). A closer look at the single-site dynamics is shown in Fig. 5 up to the time just before the propagating spirals interfere with each other at the center. Fig. 5(b) shows the comparison between the $x$-component of the local magnetization calculated with the ansatz and with t-DMRG using a Trotter time step $\delta t=0.01$ and $m=100$ (it would take too much time to sweep the exact curve). As is evident, the ansatz gives satisfactory results even for times $D t \leq 2$. For larger times, the assumption leading to Eq. (5) is no longer valid, since the perturbations have reached the third sites (measured from the chain boundaries), and three-body effects then arise. In general, as nearest-neighbor spinspin correlations propagate towards the center, manybody effects become important and the single-site dynamics deviates remarkably from what is expected from Eq. (6). As we shall discuss next in more detail, the most remarkable many-body effect is the onset of an "emergent" Rabi frequency of oscillations $\omega_{R}^{\star}$ in the single-site dynamics; these oscillations decay with time as can be anticipated from Fig. 5(a).

\section{Many-body classical dynamics}

The simplest method to investigate many-body effects in our system is by appealing to classical considerations. At the limit of commuting dynamical variables, the Heisenberg equations of motion for the spin components turn into the Landau-Lifshitz equations (without damping) for the dynamics of classical spin vectors $\boldsymbol{S}_{j}$
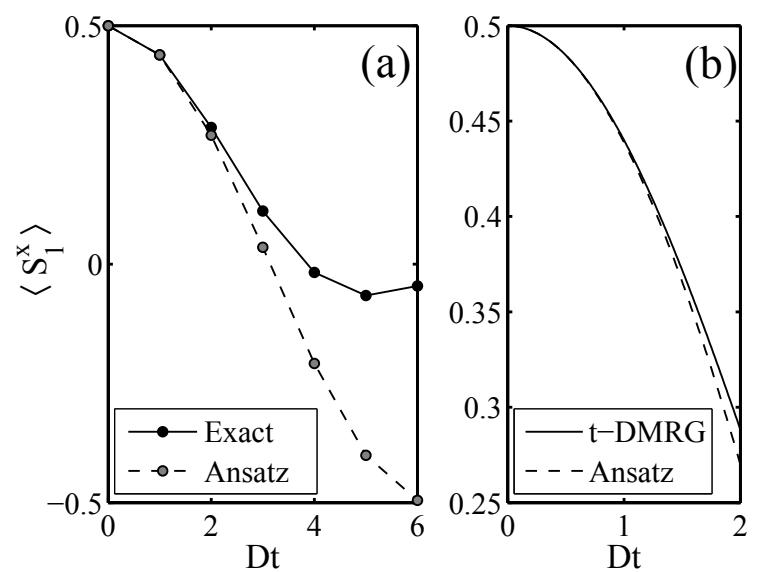

FIG. 5. Time evolution of the $x$-component of local magnetization for the first spin in a system with 14 sites. (a) Comparison between exact results with those obtained from the ansatz in Eq. (6); solid and dashed lines are guides for the eyes. (b) Finer curve obtained with t-DMRG for times $D t \leq 2$.

precessing around effective fields $\boldsymbol{B}_{j}$ :

$$
\frac{d \boldsymbol{S}_{j}}{d t}=-\boldsymbol{S}_{j} \times \boldsymbol{B}_{j}, \text { with } \boldsymbol{B}_{j}=\frac{\partial \mathcal{H}^{\mathrm{DM}}}{\partial \boldsymbol{S}_{j}} .
$$

In polar coordinates $S_{j}^{x}=\frac{1}{2} \cos \theta_{j}$, where $\theta_{j}$ is the propagating spiral profile, and if we assume $S_{j}^{y}=0$, as is evident from Figs. 2(a) and 4, we obtain the system of differential equations

$$
\dot{\theta}_{j}=\omega_{R}\left(\cos \theta_{j+1}-\cos \theta_{j-1}\right) .
$$

Let us consider the classical two-spin model first. Just after $t=0$, the first two spins are almost collinear and thus $\theta_{1} \approx \theta_{2}$. With this approximation, we have $\dot{\theta}_{1}=$ $\omega_{R} \cos \theta_{1}$ and $\dot{\theta}_{2}=-\omega_{R} \cos \theta_{2}$, with the solution

$$
2 S_{1}^{x}(t)=2 S_{2}^{x}(t)=\operatorname{sech}\left(\omega_{R} t\right) .
$$

This is plotted in Fig. 6(a) with a dash-dotted line. It suggests that the classical spin pair (decoupled from the many-body system) moves in phase, as is the case in the quantum two-spin solution. However, as may be seen from the figure, the classical dynamics of the spin pair do not display any kind of oscillation. As we shall see next, this is not the case in the many-body classical system.

The first step for solving the many-body classical dynamics is to numerically solve the system of differential equations in Eq. (8). From this system we observe that, since $\dot{\theta}_{1}=\omega_{R} \cos \theta_{2}$ and $\dot{\theta}_{N}=-\omega_{R} \cos \theta_{N-1}$, the origin of spin-spirals with opposite chiralities at the chain boundaries is a straightforward consequence of the classical open boundary conditions (see the change of signs). The solution of these equations for the first two spins along the chain is shown in Fig. [6 with thick solid lines. As can be seen, the classical solution is able to predict 


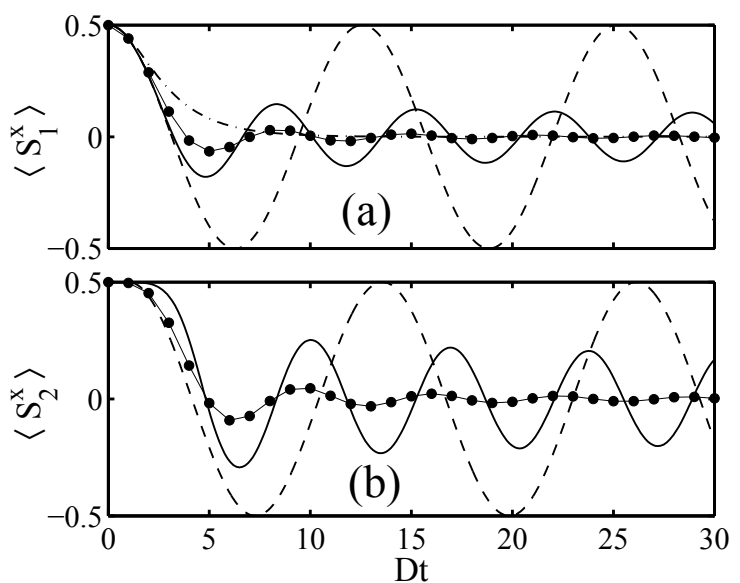

FIG. 6. Time evolution of the $x$-component of local magnetization for (a) the first spin and (b) the second spin along the chain. The black-filled circles are the results from t-DMRG. The dash-dotted line in (a) is the result of the classical twospin model described by Eq. (9). The solid line is the numerical solution from the many-body classical dynamics governed by Eq. (8), and the dashed line is the prediction from the ansatz in Eq. (6).

the decaying oscillations over time, although the decay is far slower than the data from the quantum model (results from t-DMRG). Also, the observed frequency of the oscillations $\omega_{R}^{\star}$ is very well reproduced by the classical solution. Comparing that with $\omega_{R}$ obtained from the ansatz in Eq. (6) (dashed lines), which neglects manybody correlations, we have $\omega_{R}^{\star}=2 \omega_{R}$. Thus we conclude that the Rabi oscillations in the quantum two-spin system persist in the many-body system, but with a different "emergent" frequency. This kind of behavior has appeared recently $\underline{\underline{42}}$ in the context of quantum quenches in the anisotropic spin-1/2 Heisenberg chain. There, when the initial Néel state $|\uparrow \downarrow\rangle$ is prepared and subsequently evolved under the two-spin XX Hamiltonian, Rabi oscillations (between the states $|\uparrow \downarrow\rangle$ and $|\downarrow \uparrow\rangle$ ) are observed in the staggered magnetization (antiferromagnetic order parameter) with a frequency $\omega_{R}=J$. However, when a sudden quench is performed from the many-body Néel state to the critical phase in the XX model, the order parameter still displays Rabi oscillations, but with the emergent frequency $\omega_{R}^{\star}=2 \omega_{R}$, as in the present case.

The classical single-site spin oscillations coming from the many-body numerical solution take place with the same frequency as in the quantum model, although a slight dephasing between the two results can be observed in Fig. 6. This is because the classical solution is not able to reproduce the velocity $v=a D$ with which the spin-spirals propagate (see e.g. the beginning of the corresponding curves in Fig. 6(b)). We said before that the present quantum model is equivalent to a set of free spinless fermions. In fact, the resulting dispersion relations are the same as those of the XX model, but shifted by the wave number $k_{a}=\frac{\pi}{2} a^{-1}$, since

$$
\mathcal{H}^{\mathrm{DM}}=\sum_{k} \omega_{R}^{\star} \sin (k a) c_{k}^{\dagger} c_{k}-\sum_{k} \omega_{R} e^{-i a\left(k-k_{a}\right)},
$$

where $c_{k}^{\dagger}\left(c_{k}\right)$ are creation (anihilation) operators of free spinless fermions with momentum $k$. Within the thermodynamic limit, the second term of this expression vanishes, and the group velocity of propagating excitations is given by $v_{k}=a D \cos (a k)$. Then, the propagating spinspirals can be associated with free-fermion excitations with zero total momentum (zero energy modes) giving $v_{0}=v=a D$. Hence, since this velocity comes from a purely quantum phenomenon, it is reasonable that classical considerations do not reproduce it.

In summary, we have learned from this section that the DMI term only, being exactly spin-current (quantized along the direction of $D$ ), drives the formation of spin-spirals which can be described qualitatively in classical terms, but needs input from quantum theory to fully account for the observed behavior. In particular, the two-body quantum dynamics are helpful for interpreting the more complex quantum many-body results. In the following section, we investigate how these results are modified when isotropic EC is added to the system.

\section{ADDING EXCHANGE INTERACTIONS}

In order to investigate the effects of EC on the nonequilibrium dynamics studied above, we consider some limits of the anisotropic Heisenberg exchange interaction in Eq. (11). First note that, by rotating the basis on odd (or even) sites by an angle $\pi$ around the $z$-axis, the antiferromagnetic case of the Hamiltonian $(J>0)$ can be mapped onto the ferromagnetic case $(J<0)$ with opposite sign of the anisotropy $\Delta$. In this paper we take $J>0$ as our overall energy scale (measuring time in units of $J^{-1}$ ), and give a brief overview of the quantum phase diagram as the non-dimensional parameters $\Delta$ and $D / J$ are varied. For numerical calculations we set $J=1$. The critical behavior of the (equilibrium) ground state of the model in Eq. (11) is well known ${ }^{47-49}$. For $\Delta \leq$ $-\sqrt{1+(D / J)^{2}}$, the ground state of the system is ferromagnetic. For $-\sqrt{1+(D / J)^{2}}<\Delta<\sqrt{1+(D / J)^{2}}$, the energy spectrum is gapless and the ground state does not support any kind of long-ranged order and is thus known as a spin fluid. For $\Delta>\sqrt{1+(D / J)^{2}}$, the ground state is antiferromagnetic (Néel ordered). In both the spin fluid and Néel phases, the helical spin structure in the $x y$-plane is obtained, which is one of the characteristic properties of the DMI. At the limit $\Delta \rightarrow \infty$, the Ising model with DMI is realized. The critical properties and entanglement of this model have been studied recently ${ }^{50.51}$, revealing two quantum phases: the antiferromagnetic one and the saturated chiral one, separated by the critical point $D / J=1$. At the limit $\Delta=0$, the $\mathrm{XX}$ model with DMI is obtained. Note that by rotating 


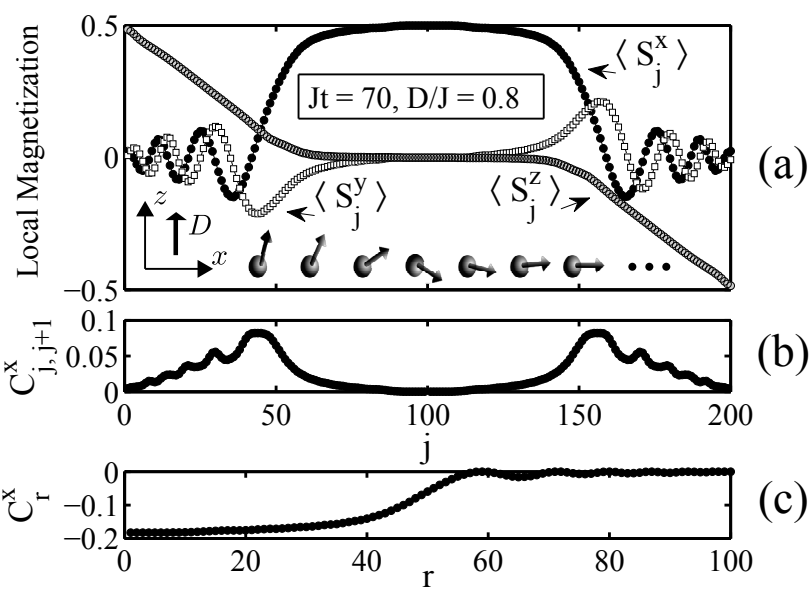

FIG. 7. (a) Snapshot of the local magnetization at the arbitrary time $J t=70$, in the model of Eq. (1) with $\Delta=1$ and $D / J=0.8$. A classical picture of the left-rotating spiral arising from the left end of the chain is drawn when $J t=2$, projected on the $x z$-plane (only the directions of spins are taken into account). (b) Nearest-neighbor spin-spin correlations. (c) Spin-spin correlations measured symmetrically from the center of the chain.

the basis at each site $j$ by an angle $(j-1) \tan ^{-1}(D / J)$ around the $z$-axis, it can be shown ${ }^{52}$ that the DMI term of the Hamiltonian can be removed, leading to a XX model with a renormalized exchange interaction energy $\sqrt{J^{2}+D^{2}}$. In the isotropic case $(\Delta=1)$, the DMI term can also be removed from the Hamiltonian ${ }^{53}$ (in fact, this can be done for any value of $\Delta$; see e.g. [47]) after a similar canonical transformation. The resulting Hamiltonian is that of the XXZ Heisenberg model

$$
\mathcal{H}^{\mathrm{XXZ}}=\sum_{j}\left[J^{x x}\left(\tilde{S}_{j}^{x} \tilde{S}_{j+1}^{x}+\tilde{S}_{j}^{y} \tilde{S}_{j+1}^{y}\right)+J^{z} \tilde{S}_{j}^{z} \tilde{S}_{j+1}^{z}\right]
$$

with planar exchange energy $J^{x x}=\sqrt{J^{2}+D^{2}}$, and anisotropy energy $J^{z}=J$. Since $J^{z} / J^{x x}<1$ for any non-zero value of $D$, the isotropic system with DMI will be in a spin-fluid phase ${ }^{49}$, as can be inferred from the discussion above.

The relaxation dynamics of the antiferromagnetic order parameter after several quantum quenches in the XXZ model has been investigated in detail recently $\underline{42}$, revealing a new mode of many-body dynamics in the effectively interacting case of finite anisotropy $\left(J^{z} / J^{x x}\right.$ in Eq. (11)): a exponential decay of the staggered magnetization with (or without) oscillatory behavior. In a mean-field approximation, a rough picture of the dynamics can be obtained by introducing pseudo-spins in $k$-space. Starting from the Néel state (equivalent to all pseudo-spins pointing in the $x$-direction), the pseudo-spins begin to precess around a $(k, t)$-dependent Zeeman field (static, and in the $z$-direction in the effectively non-interacting case of the XX model). Since the field (and hence the precession frequencies) varies continuously from pseudospin to pseudo-spin over a bandwidth, they gradually

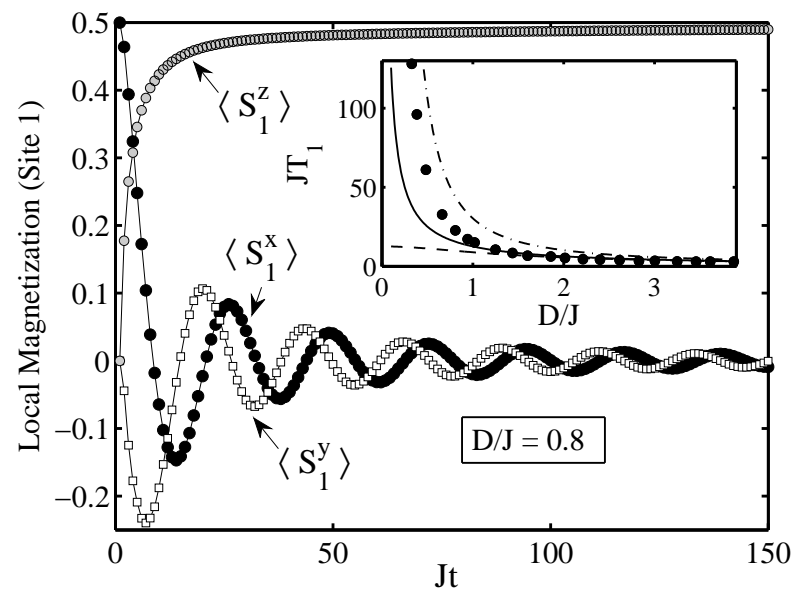

FIG. 8. Time evolution of local magnetization for the first spin along the chain for the arbitrary value $D / J=0.8$. Inset: Period of oscillations of the $x$-component of local magnetization as a function of $D / J$. The solid line is the curve $T_{R}=2 \pi / \omega_{R}$, the dashed one is the curve $T_{u}=2 \pi / \omega_{u}$, the dash-dotted line is the curve $T_{h}=2 \pi /\left(\omega_{u}-\omega_{d}\right)$, and the symbols are the results from t-DMRG.

dephase, causing the oscillations to decay with time. A similar quasi-classical picture (in real space) has been described recently in the study of coherent transport of spin currents in the spin-1/2 Heisenberg chain in transversed magnetic fields 54 . Due to the connection with a real material like $\mathrm{KCuF}_{3}$, in the following discussion, we restrict ourselves to the isotropic case $(\Delta=1)$ of the Hamiltonian in Eq. (1) (equivalent to Eq. (11)), focusing on the description of the frequency of single-site spin oscillations by means of the two-spin quantum model. We verified that, at the Ising limit $(\Delta \rightarrow \infty)$, a similar method can also approximately describe the observed behavior .

In Fig. 7 we show a snapshot of the local magnetization and spin-spin correlations in the $x$-direction at the arbitrary time $J t=70$ and $D / J=0.8$. As can be seen, inhomogeneous spin-spirals with opposite chiralities propagate again from the boundaries towards the center of the chain. As shown in the classical picture projected on the $x z$-plane when $J t=2$, the velocity of propagation is, in general, greater than in the case with $J=0$. As expected, the presence of EC promotes the onset of strong long-ranged spin-spin correlations (see Fig. 7(c)). However, these correlations seems to be large for spin pairs (with respect to the center of the chain) that have not been touched by the spiral perturbations and diminish strikingly to zero for those that have undergone single-site oscillatory dynamics for a considerable time; that is, the propagating spin-spirals with opposite chiralities are almost uncorrelated. We also observe from Fig. $7(b)$ that the nearest-neighbor spin-spin correlations are strongly weakened compared to the case with $J=0$, going to negligible values at (and near) the chain boundaries. A remarkable feature from Fig. 77(a) is that in the presence of $\mathrm{EC}$, the $y$-component of local magne- 
tization is no longer "quenched", as it was in the case with $J=0$. A closer look at the single-site quantum dynamics is shown in Fig. 8, where the time evolution of local magnetization for the first site along the chain is calculated. We immediately see that beginning in the state $|\rightarrow\rangle$, this spin precesses (or "spirals") clockwise towards the asymptotic state $|\uparrow\rangle$ with a frequency which we shall investigate next. We verified that the decay of oscillations is exponential. At the other boundary, the spin precesses counterclockwise towards the asymptotic state $|\downarrow\rangle$ with the same frequency. These perturbations are transmitted to the spins in the bulk apparently in a diffusive way, in contrast to the ballistic propagation which is observed in the case with $J=0$.

Based on the experience of the former section, we may want to consider the two-spin quantum dynamics of the model with DMI and isotropic EC. In Fig. 3(b) we show the energy diagram with $\omega_{d}=J / 2$ and $\omega_{u}=\sqrt{\omega_{R}^{2}+\omega_{d}^{2}}$. In this case, the energy gap between the ground and first excitated state (which remains two-fold degenerate) is larger than in the case with $J=0$. The $x$-component of local magnetization of the spin par oscillate in phase

$$
2\left\langle S_{1}^{x}\right\rangle=\cos \left(\omega_{d} t\right) \cos \left(\omega_{u} t\right)+\frac{\omega_{d}}{\omega_{u}} \sin \left(\omega_{d} t\right) \sin \left(\omega_{u} t\right) .
$$

The nature of these oscillations depends strongly on the ratio $\omega_{d} / \omega_{u}={\sqrt{1+(D / J)^{2}}}^{-1}$ which coincides with the anisotropy $J^{z} / J^{x x}$ in Eq. (11). Then, when $D / J \gg 1$ the leading behavior is governed by the first term of the sum in Eq. (12). This term describes fast oscillations with period $T_{u}=2 \pi / \omega_{u}$ modulated by the cosine curve $\cos \left(\omega_{d} t\right)$, i.e, a beat-like pattern. According to Eq. (11), in this regime, the many-body system tends to be described by the XX model and we expect that these fast oscillations will resemble the Rabi oscillations found in the former section. To test these predictions, we calculate the period of oscillations (by averaging the time between sequential troughs) for the first spin along the chain as a function of $D / J$, using t-DMRG. This is shown in the inset of Fig. 8. There, we see how the dashed line (representing $T_{u}$ ) tends to coincide with the solid one (representing $T_{R}=2 \pi / \omega_{R}$ ) in the regime $D / J \gg 1$, and both curves well describe the actual period of the single-site oscillations in the many-body system. On the other hand, when $0<D / J \ll 1$, the behavior in Eq. (12) is approximately well described by the curve $\cos \left[\left(\omega_{u}-\omega_{d}\right) t\right]$ which displays oscillations with the long period $T_{h}=2 \pi /\left(\omega_{u}-\omega_{d}\right)$. This is represented in the inset of Fig. 8 by the dash-dotted line. At the limit $D / J \rightarrow 0$ (isotropic Heisenberg model), the spin oscillations are not expected to be observed, as inferred by the divergence of the period of oscillations. In fact, our initial spin-polarized state in the $x$-direction is a stationary state of this model and hence no time evolution is detected in local observables, as we have verified numerically. It is curious to note that the period of spin oscillations in the two regimes can be remembered easily if we make an analogy with a radiative electronic transition. It

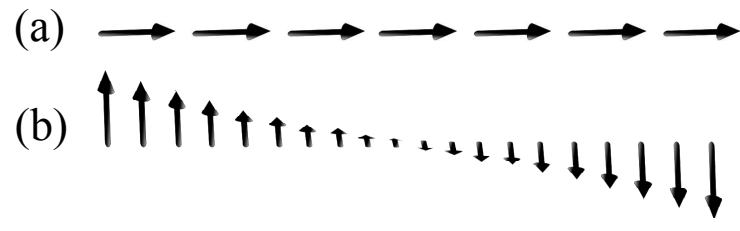

FIG. 9. (a) Initial spin-polarized state in the $x$-direction. (b) Approximate classical picture of the appearance of the state after the spin-spirals with opposite chiralities interfere at the center of the chain (see the text for deviations).

is well known from elementary quantum mechanics that if an electron is undergoing a transition between stationary states $n$ and $l$, its position oscillates sinusoidally with an angular frequency $\left(E_{n}-E_{l}\right) / \hbar$. Thus, in the regime $D / J \gg 1$, we might think of the spins as "undergoing" radiative transitions between the ground and first excited state (see Fig. 3(b)) and hence oscillating with the pe$\operatorname{riod} T_{<} \approx 2 \pi /\left(\omega_{u}+\omega_{d}\right)$, and in the regime $0<D / J \ll 1$, they might be thought of as undergoing radiative transitions between the first and highest excited state and hence oscillating with the period $T_{>} \approx 2 \pi /\left(\omega_{u}-\omega_{d}\right)$.

One may ask about the validity of using the quantum two-spin model to interpret results from the manybody system in the present case. In the former section we argued that in the absence of EC this can be done for a certain time interval, since long-ranged spin-spin correlations were almost "turned off" during the spiral propagation and only the transport of nearest-neighbor spin-spin correlations were responsible for the onset of quantum many-body effects, which make the predictions from the quantum two-spin model fail. In the presence of EC, we see that strong long-ranged spin-spin correlations develop in the system, and yet the single-site spin oscillations can be described approximately well by the quantum two-spin model. We believe that this happens due to the fact that only nearest-neighbor spin-spin correlations carry the information of relevant many-body effects and these correlations are very small in the presence of EC, especially at the boundaries (see Fig. 7(b)). As noted from the inset of Fig. 8, this picture is very suggestive, since there are no "emergent" Rabi frequencies in the presence of EC; i.e., the frequencies predicted by the quantum two-spin model are those approximately observed in the single-site dynamics of the many-body system. This was not the case in the absence of EC, since nearest-neighbor spin-spin correlations were remarkably larger during the spiral propagation.

\section{COMMENTS ON THE LONG-TIME DYNAMICS}

In the previous sections, we studied the short-time dynamics of the spin-spiral propagation and oscillations in a spin chain with DMI and with(out) EC. By this, we always meant the time evolution just after the quench and 
before the two perturbations coming from the boundaries interfere at the center of the chain. Even though we are not going to go deeper into what happens after this, it is worthwhile saying something for the sake of completeness. As might be expected intuitively, when the spinspirals with opposite chiralities encounter one another, complex interference patterns arise. We observed in all cases that the components of local magnetization transverse to the direction of the DMI take on very small values as each propagating perturbation goes across the opposite half of the chain from where it was created, which is reasonable, due to the "destructive" character of interference in the $x y$-plane: spins in different halves of the chain have opposite direction of rotation (when they precess) in the $x y$-plane before the propagation, and almost uncorrelated spin-spirals reach the center. Then, beginning with our initial spin-polarized state in the $x$ direction as depicted in Fig. 9(a), we arrive after a long time at a state with an appearance approximately similar to that shown classically in Fig. 9(b), but with small fluctuations in the $x y$-plane which support the propagation of residual spin waves. Since we could not access very long times with our numerical calculations, it was not possible to study properties in the very long-time limit.

\section{CONCLUSIONS}

We have investigated the non-equilibrium dynamics of a spin-1/2 chain with Dzyaloshinskii-Moriya interactions in the presence (and absence) of Heisenberg exchange interactions after a quench by a transverse field. We found in all cases that inhomogeneous spin-spirals with opposite chiralities propagate from the boundaries towards the center of the chain until they meet at the center, producing complex interference patterns. This propagation is accompanied by quantum spin oscillations that decay asymptotically over time. We showed that important information for the interpretation of the observed quantum behavior can be extracted from classical considerations of the spin system, as well as from the quantum two-spin model of interactions. Our results contribute to recent efforts to understand the characteristics of the non-equilibrium dynamics following a quantum quench in closed one-dimensional spin systems, and are expected to be helpful for possible potential applications where the Dzyaloshinskii-Moriya interaction plays a relevant role in low-dimensional systems.

\section{ACKNOWLEDGMENTS}

The author E.S.C. thanks the support from the Fulbright-Colciencias fellowship and the Mazda Foundation for the Arts and the Science fellowship. The support from the Dirección de Investigaciones Sede Bogotá of the Universidad Nacional de Colombia is also acknowledged.
1 A. Polkovnikov et al., arXiv: 1007.5331v1 (2010).

2 I. Bloch et al., Rev. Mod. Phys. 80, 885 (2008).

3 L.-M. Duan et al., Phys. Rev. Lett. 91, 090402 (2003).

4 S. Trotzky et al., Science 319, 295 (2008).

5 J. Simon et al., Nature 472, 307 (2011).

${ }^{6}$ V. Mukherjee et al., Phys. Rev. B 76, 174303 (2007).

7 V. Mukherjee and A. Dutta, J. Stat. Mech. p. P05005 (2009).

8 K. Sengupta and D. Sen, Phys. Rev. A 80, 032304 (2009).

${ }^{9}$ H. L. Guo et al., Eur. Phys. J. B 79, 503 (2011).

10 D. Rossini et al., Phys. Rev. Letts. 102, 127204 (2009).

11 P. Calabrese et al., arXiv: 1104.0154v1 (2011).

12 P. Calabrese and J. Cardy, J. Stat. Mech. p. P04010 (2005).

13 A. Das et al., Phys. Rev. B 74, 144423 (2006).

14 Y. Li et al., Phys. Rev. B 80, 054404 (2009).

15 M. Rigol et al., Phys. Rev. Lett. 98, 50405 (2007).

16 M. Rigol et al., Nature 452, 854 (2008).

17 C. Bennett and D. DiVincenzo, Nature 404, 247 (2000).

18 M. Greiner et al., Nature 419, 51 (2002).

19 S. Hofferberth et al., Nature 449, 324 (2007).

20 D. Awschalom and N. Samarth, Physics 2, 50 (2009).

21 L. Dreher et al., Phys. Rev. Lett. 106, 037601 (2011).

22 B. W. Lovett, Physics 4, 6 (2011).

23 I. Dzyaloshinsky, J. Phys. Chem. Solids 4, 241 (1958).

24 T. Moriya, Phys. Rev. 120, 91 (1960).

25 M. Bode et al., Nature (London) 447, 190 (2007).

26 P. Ferriani et al., Phys. Rev. Lett. 101, 027201 (2008).
27 S. Meckler et al., Phys. Rev. Lett. 103, 157201 (2009).

28 T. Kimura et al., Phys. Rev. Lett. 94, 137201 (2005).

${ }^{29}$ G. Lawes et al., Phys. Rev. Lett. 95, 087205 (2005).

30 M. Kenzelmann et al., Phys. Rev. Lett. 95, 087206 (2005).

31 Y.-J. Lin et al., Nature 471, 83 (2011).

32 I. Yamada et al., J. Phys.: Condens. Matter 1, 3397 (1989).

33 M. Kargarian et al., Phys. Rev. A 79, 042319 (2009).

34 T. Yu and J. Eberly, Phys. Rev. Lett. 93, 140404 (2004).

35 Z. H. Fang et al., Chin. Phys. B 18, 3265 (2009).

36 J. L. Guo and H. S. Song, Eur. Phys. J. D 56, 265 (2010).

37 S. R. White and A. E. Feiguin, Phys. Rev. Lett. 93, 076401 (2004).

38 A. J. Daley et al., J. Stat. Mech. p. P04005 (2004).

39 T. Antal et al., Phys. Rev. E 59, 4912 (1999).

40 V. Hunyadi et al., Phys. Rev. E 69, 066103 (2004).

41 D. Gobert et al., Phys. Rev. E 71, 036102 (2005).

42 P. Barmettler et al., New J. Phys. 12, 055017 (2010).

43 E. H. Lieb and D. W. Robinson, Commun. Math. Phys. 28, 251 (1972).

44 P. Calabrese and J. Cardy, Phys. Rev. Lett. 96, 136801 (2006).

45 S. R. White, Phys. Rev. B 48, 10345 (1993).

46 S. R. Manmana et al., Phys. Rev. B 79, 155104 (2009).

47 F. C. Alcaraz and W. F. Wreszinski, J. Stat. Phys. 58, 45 (1990).

48 A. A. Zvyagin, J. Phys.: Condens. Matter 3, 3865 (1991).

49 R. Jafari and A. Langari, arXiv: 0812.1862v1 (2008). 
50 R. Jafari et al., Phys. Rev. B 78, 214414 (2008).

51 C. T. Gonzalez-H et al., Eur. J. Phys. 31, 681 (2010).

52 O. Derzhko and T. Verkholyak, Proc. Inst. Math. NASU 50, 692 (2004)
53 D. N. Aristov and S. V. Maleyev, Phys. Rev. B 62, R751 (2000).

54 R. Steinigeweg et al., Phys. Rev. Lett. 106, 160602 (2011). 